\title{
Economía de las energías eólicas
}

\author{
Benito Vera \\ Director General de 4 innovation
}

\section{Resumen}

La potencia eléctrica eólica mundial alcanzó 238 GW en 2011, por lo que podemos considerar que ya se ha convertido en una tecnología mainstream. En los últimos 10 años sus costes de producción han ido progresivamente bajando hasta niveles de entre 52 y $70 \square / M W h$ en términos del coste normalizado de la energía (LCOE) según distintos estudios, que la hacen prácticamente competitiva con alternativas convencionales. El futuro desarrollo del sector pasa, además de seguir incrementando en áreas geográfica poco penetradas, por el desarrollo de la tecnología eólica marina, sobre la que Europa y China están haciendo una fuerte apuesta.

Palabras clave: energía eólica terrestre, energía eólica marina, marcos regulatorios.

Clasificación JEL: Q42, Q48.

\begin{abstract}
After reaching 238 GW world-installed capacity in 2011, wind-power can be considered a mainstream electricity generation technology today. Over the last 10 years significant cost reductions have been achieved, showing estimated production costs (LCOE) of $\square 52-70$ per MWh, almost competitive now with other conventional generation technologies. Wind's future will be driven by growth in new geographical areas lightly penetrated today and the development of offshore wind capacity, strongly focused in Europe and China.
\end{abstract}

Keywords: wind onshore generation, wind offshore generation, regulatory frameworks.

JEL classification: $Q 42, Q 48$.

\section{Introducción}

En este artículo hacemos balance sobre la situación actual y perspectivas de la energía eólica, tras una década de decidido crecimiento en el número de instalaciones. Para ello analizamos el momento actual de costes y nivel de competitividad, los modelos y papel de los marcos regulatorios en esta dinámica de expansión y finalizamos con algunas apreciaciones sobre el emergente campo de la tecnología eólica marina.

\section{Photo finish: situación actual y balance de la energía eólica terrestre}

A finales de 2011 la potencia instalada eólica mundial alcanzaba los 238,3 GW con un crecimiento del 20,6 por 100 respecto al año anterior, equivalente a 41,2 GW 
de nueva instalación ese año, de acuerdo con los datos provisionales publicados en febrero por el Global Wind Energy Council ${ }^{1}$ (GWEC).

La distribución geográfica de esta potencia instalada muestra una gran concentración en tres regiones (Europa, Norteamérica y Asia) representando el 97 por 100 del total.
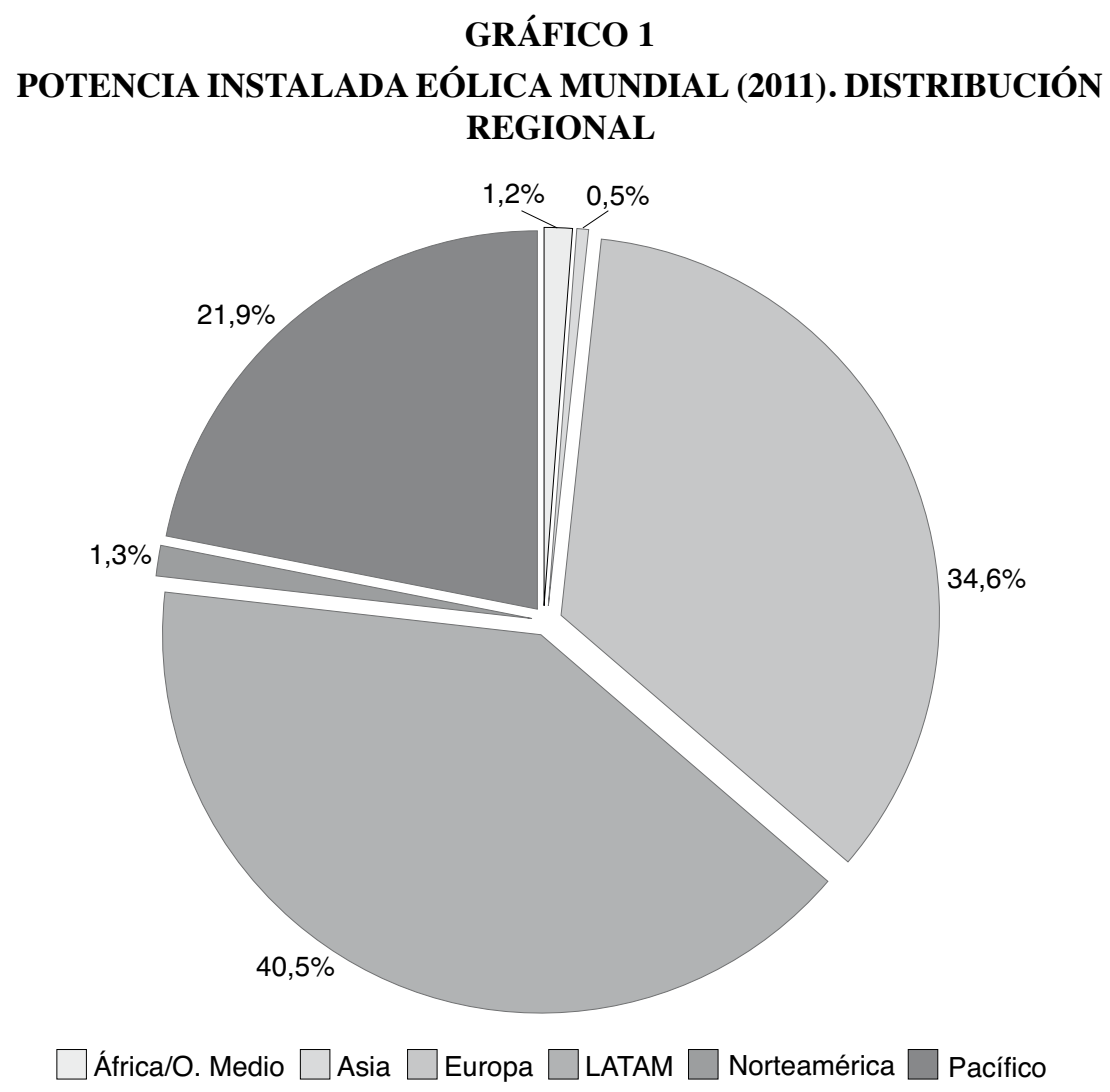

FUENTE: GWEC (2012).

Significativamente, en China (un gigante en todos los ámbitos económicos) se contabilizan 62,7 GW de los 82,4 GW instalados en la región asiática lo que le coloca también en líder mundial por potencia instalada eólica.

\footnotetext{
${ }^{1}$ GWEC (2012): Global Wind Statistics, 2011.
} 
CUADRO 1

POTENCIA INSTALADA EÓLICA (2011). TOP 10 PAÍSES

\begin{tabular}{|l|c|c|}
\hline & $\begin{array}{c}\text { Potencia instalada } \\
\text { GW }\end{array}$ & $\begin{array}{c}\text { Total } \\
\mathbf{\%}\end{array}$ \\
\hline China & 62,7 & 26,3 \\
\hline Estados Unidos & 46,9 & 19,7 \\
\hline Alemania & 29,1 & 12,2 \\
\hline España & 21,7 & 9,1 \\
\hline India & 16,1 & 6,7 \\
\hline Francia & 6,8 & 2,9 \\
\hline Italia & 6,7 & 2,8 \\
\hline Reino Unido & 6,5 & 2,7 \\
\hline Canadá & 5,3 & 2,2 \\
\hline Portugal & 4,1 & 1,7 \\
\hline
\end{tabular}

FUENTE: GWEC (2012).

Detrás de esta foto del sector, se enmascaran dos tendencias: una fuerte aceleración en el desarrollo de la potencia eólica en la segunda mitad de la última década y un proceso de relevo entre regiones en el tiempo.

Respecto a la primera tendencia, 2005 es un año muy relevante para la industria: la instalación anual de aerogeneradores superó $11 \mathrm{GW}$ por primera vez, lo que contrasta, por ejemplo, con los 7,5 GW de media anual registrados en el periodo 2000-2004. Es más, a partir de ese momento se registra una aceleración sostenida en las cifras de instalación anual de potencia, a pesar de la crisis económica desde 2008, hasta alcanzar el record en 2011 con 41,2 GW como se ha mencionado anteriormente. 


\section{GRÁFICO 2}

INSTALACIÓN ANUAL DE POTENCIA EÓLICA (MW) (1996-2011). TOTAL MUNDO

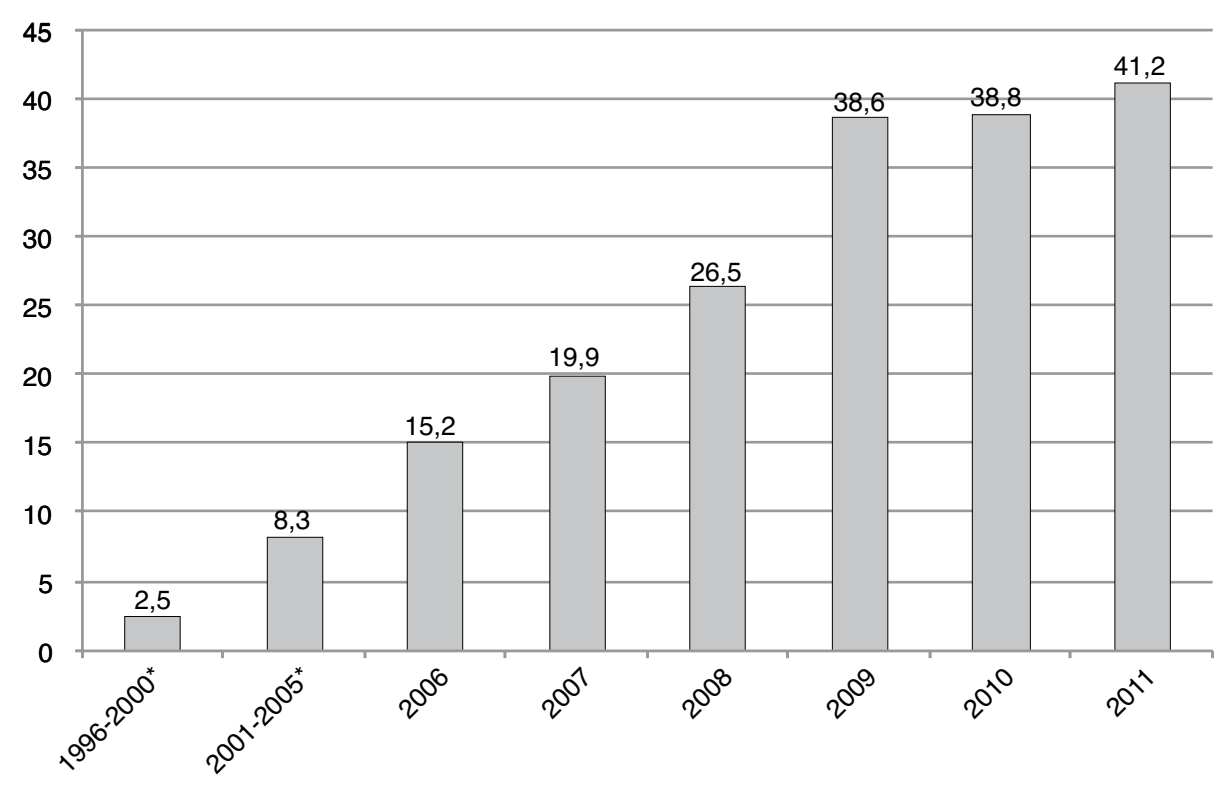

NOTAS: * Medias anuales.

FUENTE: GWEC (2012).

Respecto a la segunda tendencia, se constata una rotación geográfica en el liderazgo del desarrollo eólico. Si en las épocas tempranas (primera mitad de la pasada década) países europeos como Alemania o España se sitúan claramente en cabeza por número de instalaciones anuales, Estados Unidos se incorpora a la carrera con gran fuerza a partir de 2006-2007, en tanto el gigante chino despierta en 2008. 


\section{GRÁFICO 3}

INSTALACIÓN ANUAL DE POTENCIA EÓLICA (MW) (2001-2011).

\section{PAÍSES SELECCIONADOS}

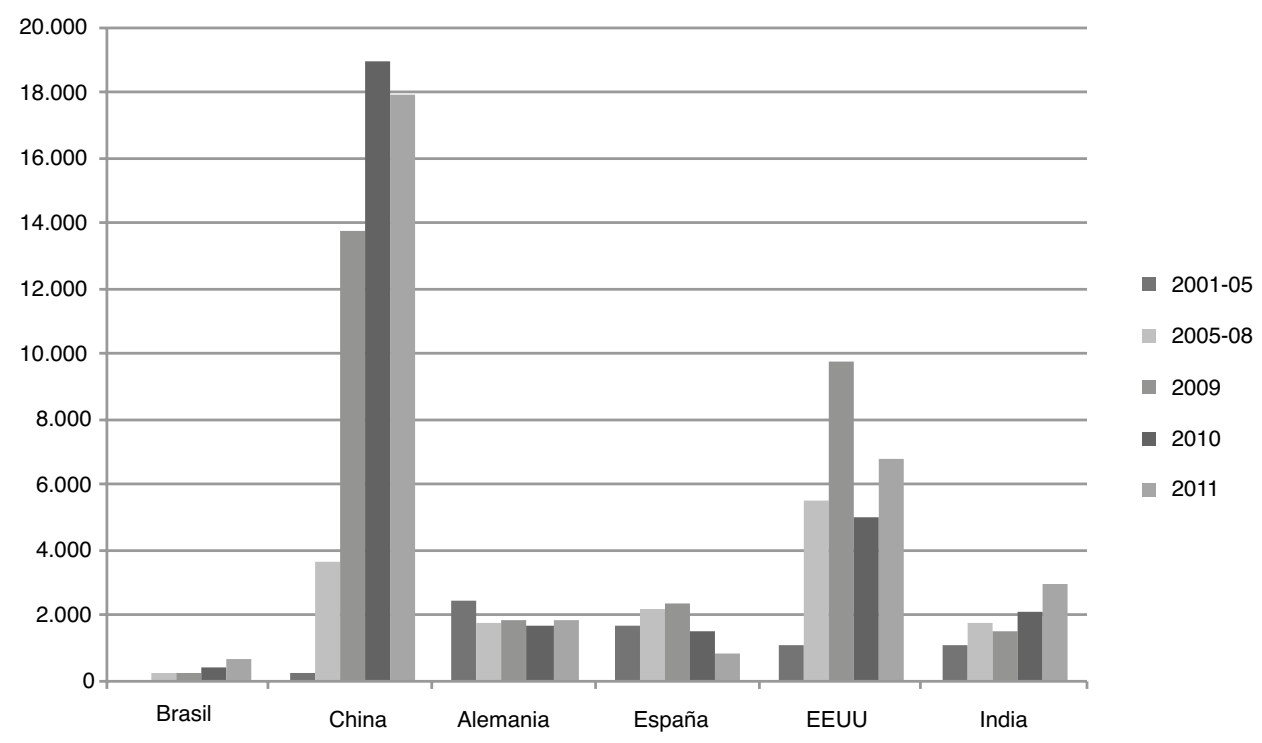

Fuente: GWEC (2012).

Los últimos tres años (2009-2011) han visto también el despertar de otras dos potencias económicas emergentes (India y Brasil), que al día de hoy se consideran los mercados del futuro por la mayoría de los analistas del sector.

\section{MODELOS REGULATORIOS}

Aunque tratado de forma más extensa en otros artículos de este especial, empecemos por repasar brevemente las razones que justifican la necesidad de marcos regulatorios de apoyo al desarrollo de renovables.

En líneas generales, esta necesidad surge de:

- La existencia de externalidades económicas en la producción de energía eléctrica con otras fuentes (fósiles habitualmente) y ligadas a aspectos medioambientales, que el uso de tecnologías renovables evitan.

- La contribución a los objetivos de la política energética, como por ejemplo la independencia energética (uso de fuentes autóctonas), o de política económica (apoyo a la creación de empleo o inversión en I+D).

- La distinta naturaleza económica de la inversión en las distintas tecnologías de generación: las renovables se caracterizan por altos costes fijos, y deben competir en desigualdad con otras tecnologías (por ejemplo, ciclos combinados), 
que fijan los precios marginales de la electricidad y que disfrutan de muy bajos costes fijos a su vez.

Existe un gran número de mecanismos específicos de promoción de las energías renovables. La siguiente tabla ofrece un resumen esquemático de las diferentes tipologías existentes.

\section{CUADRO 2}

TIPOLOGÍA DE SISTEMAS DE APOYO A LAS ENERGÍAS RENOVABLES

\begin{tabular}{|c|c|c|c|c|}
\hline & \multicolumn{2}{|c|}{ Directo } & \multirow{2}{*}{ Indirecto } \\
\hline & & Precio & Capacidad & \\
\hline \multirow[t]{2}{*}{ Regulado } & $\begin{array}{l}\text { Orientado a la } \\
\text { inversión }\end{array}$ & $\begin{array}{l}\text { - Subsidios } \\
\text { - Crédito fiscal }\end{array}$ & - Subastas & $\begin{array}{l}\text { - Impuestos } \\
\text { ambientales }\end{array}$ \\
\hline & $\begin{array}{l}\text { Orientado a la } \\
\text { producción }\end{array}$ & $\begin{array}{l}\text { - Feed-in-Tariffs } \\
\text { - Primas }\end{array}$ & $\begin{array}{l}\text { - Subastas } \\
\text { - Certificados } \\
\text { verde }\end{array}$ & \\
\hline Voluntario & $\begin{array}{l}\text { Orientado a la } \\
\text { inversión }\end{array}$ & $\begin{array}{l}\text { Donaciones, } \\
\text { ayuda al } \\
\text { desarrollo } \\
\text { Tarifas verdes }\end{array}$ & & $\begin{array}{l}\text {-Acuerdos } \\
\text { voluntarios }\end{array}$ \\
\hline
\end{tabular}

FUENTE: EWEA (2005).

Se distinguen en primer lugar entre mecanismos de apoyo directo e indirecto. Los primeros buscan un impacto inmediato sobre el volumen de capacidad instalada, en tanto los segundos están orientados a crear las condiciones necesarias para el desarrollo de la inversión en el medio/largo plazo.

Un segundo conjunto de criterios referencia a la obligatoriedad o voluntariedad de cumplimiento, estando los segundos (voluntarios) basados en unos compromisos asumidos por parte de algunos agentes, no necesariamente ligados a incentivos económicos específicos (por ejemplo, consumidores dispuestos a pagar precios diferentes por el disfrute de energía de origen «verde»)

Finalmente una distinción en función de la meta a la que se dirige el mecanismo, pudiendo estar orientado bien a fijar unos precios compatibles con unos niveles de inversión indicativos, o por el contrario, ir directamente a fijar unos niveles de potencia instalada/producción pero renunciando al control de los precios.

Los mecanismos regulados orientados al precio y a la inversión son utilizados fundamentalmente en los EEUU. Son mecanismos más complejos que el resto, basados en exenciones fiscales sobre beneficios, deducciones por la inversión y/o esquemas de amortización fiscal acelerada de los activos. En estos esquemas, con frecuencia el promotor de las inversiones es incapaz de aprovechar al 100 por 100 estos beneficios, por insuficiencia de sus bases fiscales, lo que obliga a la búsqueda de socios financieros (tax equity investors) a los que revender dichas deducciones ${ }^{2}$.

\footnotetext{
2 Véase una interesante reflexión sobre su eficiencia económica en SCHMALENESEE (2011)
} 
Por su lado, los mecanismos regulados orientados a la producción y el precio constituyen el modelo más extendido en Europa. Estos tratan de compensar el valor de la «externalidad» no recogido por los precios de mercado, ofreciendo una renta extra al productor renovable, de manera que todas las tecnologías compitan en igualdad de condiciones (level playing field).

Las dos formas más comunes son los sistemas de primas y los denominados feedin-tariffs. Los primeros consisten en unos pagos fijos al generador (componente «verde») que se añaden al precio de mercado (componente «marrón») y pueden ir acompañados de medidas complementarias, como por ejemplo:

- Suelos y techos de retribución total, buscando garantizar unos rangos de rentabilidad razonables al inversor en las tecnologías, al tiempo que limitar el coste total para los consumidores.

- Obligaciones de compra de toda la energía vertida de origen renovable por un agente (por ejemplo, el operador del mercado).

Los feed-in-tariffs (FiTs) son tarifas fijas establecidas de forma administrativa, lo que garantiza al productor un flujo de ingresos recurrentes e independiente de la volatilidad de los precios del mercado.

Los mecanismos regulados a la capacidad y a la inversión tienen su desarrollo más conocido en los sistemas de subastas competitivas, y en Brasil a uno de los casos de éxito más reciente.

Los mecanismos regulados a la capacidad y a la producción se basan en la emisión de unos certificados de carácter verde o renovable (lado de la oferta), complementado por una obligación de compra (lado de la demanda) por algún agente del sistema, normalmente las distribuidoras o las comercializadoras eléctricas. El ingreso obtenido por la venta de estos certificados se suma al precio de mercado de la energía, y ambos conforman el ingreso total de los productores.

Debe destacarse que en este sistema, debido a la existencia de unas obligaciones de compra, normalmente crecientes en el tiempo, suele haber un importante incentivo a la firma de acuerdos de suministro a largo plazo (PPAs), lo que da un elevado nivel de certidumbre de sus ingresos al generador.

Este modelo ha sido abrazado en el Reino Unido (sistema de Renewable Obligation Certificates o ROCs) y en un gran número de estados de los EEUU (Renewable Portfolio Standards o RPSs).

¿Existe algún modelo mejor o superior a los demás? La evidencia no es concluyente y además ningún país ha adoptado un modelo puro, sino que normalmente encontramos combinaciones de mecanismos.

A pesar de ello, hay una relativa mayor orientación hacia los esquemas regulatorios basados en FiTs o primas, con resultados muy positivos, como lo demuestra el importante impulso al desarrollo de renovables en Europa Continental frente a Reino Unido, por ejemplo, que ha implementado un sistema basados en certificados. 
Incidentalmente, es importante señalar que en julio de 2011 el gobierno británico planteó un proyecto encaminado a reformar su mercado energético ${ }^{3}$, ante la urgencia de invertir en nuevas infraestructuras y con el objetivo de apoyar los esfuerzos de descarbonización de la economía.

El pilar de la reforma está constituido por una serie de medidas en apoyo de la inversión en generación eléctrica de bajo o nulo contenido de $\mathrm{CO}_{2} \mathrm{y}$, en lo tocante a las renovables, la introducción de un sistemas de FiTs con contratos por diferencias; éste sustituiría al actual marco basado en ROCs. La justificación para este cambio estriba en el análisis realizado por el gobierno, que concluye en una falta de eficacia del actual marco regulatorio para afrontar el importante reto inversor en energías renovables al que necesita enfrentarse el país, y por tanto en la necesidad de crear un marco más potente.

Ahora bien, en contra de este argumento sobre la superioridad de esquemas basados en FiTs o primas se puede poner el ejemplo de Estados Unidos, que tiene un sistema de incentivos fiscales federal y de certificados estatales. En el último lustro ha experimentado un desarrollo acelerado en la instalación de nueva capacidad renovable (eólica y solar), hasta situarse en el segundo país del mundo por capacidad instalada eólica justo detrás de China.

Sin embargo no podemos tampoco obviar el muy favorable impacto que han tenido sobre la inversión en energías renovables en Norteamérica, los apoyos directos a la inversión que la administración Obama implementó al comienzo de su mandato, precisamente como respuesta a la insuficiencia del esquema tradicional de apoyos fiscales, en un contexto de fuerte crisis económica y de contracción de la financiación bancaria y de los mercados de capitales.

\section{Coste y competitividad de la energía eólica terrestre}

Distinguiremos en este apartado entre dos conceptos tradicionales de la economía, como son el de coste de producción y el de competitividad de dicha producción en referencia a otras fuentes de energía, haciendo además algunas consideraciones sobre las externalidades en la actividad energética, que pueden llevar a apreciaciones erróneas sobre este último concepto.

El indicador para el coste de producción más ampliamente utilizado por los expertos del sector es el denominado levelised cost of energy (LCOE), normalmente traducido como «coste normalizado de la energía».

Su utilidad para este tipo de ejercicios viene explícitamente avalada por la Agencia Internacional de la Energía (AIE) ${ }^{4}$ cuando lo califica de «herramienta útil para comparar los costes unitarios de diferentes tecnologías a lo largo de su vida útil económica» ${ }^{5}$.

\footnotetext{
${ }^{3}$ http://www.decc.gov.uk/en/content/cms/legislation/white_papers/emr_wp_2011/emr_wp_2011.aspx.

4 AIE (2011): Projected Costs of Generating Electricity, 2010 Edition, pp. 33 y ss.

5 «handy tool for comparing the unit costs of different technologies over their economic life».
} 
El LCOE, definido como coste unitario de producción ( $€ / \mathrm{kWh})$ es el resultado de un árbol de magnitudes financieras, de costes y operativas de una instalación eólica, interrelacionados entre sí, a lo largo de la vida útil del proyecto, y comprendería los siguientes componentes ${ }^{6}$ :

\section{Costes monetarios anuales.}

a) Costes de capital anuales $(€)$.

I. Costes de inversión $(€)$.

II. Vida útil de la instalación (años).

III. WACC (coste medio ponderado del capital) del proyecto (\%).

b) Costes de operación y mantenimiento anuales $(€)$.

\section{Producción anual de energía.}

a) Características técnicas de la turbina (altura, diámetro rotor, etcétera.).

b) Características del emplazamiento (recurso eólico, velocidad de los vientos, etcétera.).

El informe preparado por la European Wind Energy Association en 2009 revela un rango de costes para la producción de eólica de 50-100 €/MWh y una media de $70 \square / M W h$ (precios de 2006) con claras diferencias entre zonas de bajo recurso eó$\operatorname{lico}^{7}$ (70-100 €/MWh) y zonas de alto recurso 8 (50-65 €/MWh).

Por otro lado, un muy reciente estudio de Bloomberg New Energy Finance (BNEF), correspondiente al segundo trimestre de $2011^{9}$, muestra una significativa mejora del rango al situarlo entre aproximadamente $45-85 € / \mathrm{MWh}^{10}$ y una media de unos 52 $\square / M W h$, a precios corrientes de 2011 .

Obviamente la comparativa no es homogénea, afectada por la utilización de tasas de descuento o escandallo de costes (turbinas, por ejemplo) diferentes. Pero sí marca una tendencia que, junto con alguna otra evidencia reciente ${ }^{11}$, permite prever que nos encontramos en la antesala de que la energía eólica terrestre haya entrado en la zona de plena competitividad ${ }^{12}$.

¿Qué factores (drivers) están impulsando esta reducción de los costes de producción de electricidad de origen eólico?

Empecemos por entender la naturaleza económica de los proyectos eólicos ya que una de sus principales característica es la gran importancia del componente inversión, que representa en torno al 75 por 100 del coste total de la energía. Ello

\footnotetext{
${ }^{6}$ Página 30 de EWEA (2009): The Economic of Wind Energy.

71500 a 1.900 horas anuales

8 2.700-2.900 horas anuales.

${ }^{9}$ Citado por GAMESA en su presentación Spain Investor Day: Energía Eólica: la energía sostenible líder (11 de enero de 2012).

${ }^{10}$ Datos originales del estudio expresados en US\$, convertidos a un tipo de cambio de 1,3 US\$/€.

${ }^{11}$ En las subastas competitivas de nueva generación organizadas en Brasil, los precios ofertados por productores eólicos se han situado por primera vez en niveles inferiores a los de operadores de ciclos combinados. Nótese no obstante, que se trata de emplazamientos de recurso eólico excepcional (por encima de 3.000 horas anuales).

${ }^{12}$ En una reciente intervención pública, Jorge Calvet, presidente de Gamesa, anticipaba que para 2016 la eólica competiría ya en igualdad de condiciones con otras alternativas convencionales.
} 
contrasta con la estructura de costes de las plantas de ciclo combinado de gas, por ejemplo, en las que el componente inversión puede representar entre el 30 por 100 y el 60 por 100 dependiendo del régimen de funcionamiento de la mismas (peak o base load), siendo el resto básicamente combustible y en menor medida los gastos de operación y mantenimiento (O\&M).

Este coste de inversión se subdivide a su vez en diversos componentes, siendo la turbina el capítulo más importante (76 por 100) seguido por las infraestructuras para conexión en red (9 por 100) y cimentación (6 por 100).

\section{GRÁFICO 4 \\ COSTES DE INVERSIÓN DE UNA TURBINA EÓLICA*}

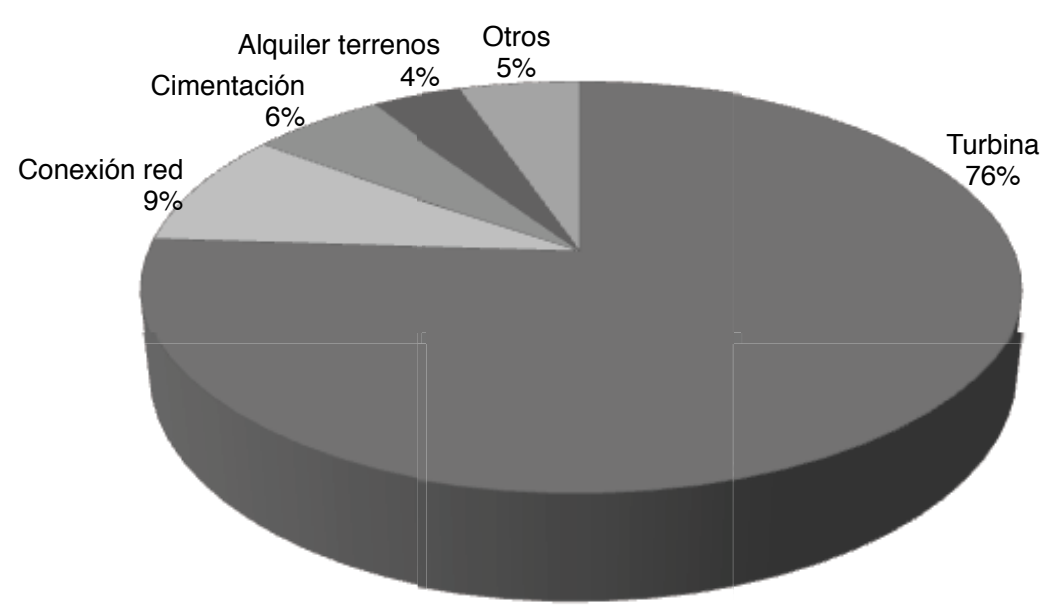

NOTA: * Modelo 2 MW instalado en Europa (datos 2006)

FUENTE: EWEA (2009).

Los restantes componentes de los costes de generación (25 por 100 en media) tienen su origen en los gastos de operación y el mantenimiento (O\&M) que según los datos del estudio de EWEA se situaban en un rango de 12-15 €/MWh. De éstos, un 60 por 100 aproximadamente son estrictamente O\&M (repuestos y mano de obra), y el restante 40 por 100 comprende seguros, gastos generales y otros.

La tendencia a la baja experimentada en estos años por los costes de generación eólica es un ejemplo casi de libro tanto de la teoría de las economías de escala en la producción, como de la aplicación de curvas de aprendizaje.

Por poner en contexto estas mejoras, los datos del informe de BNEF citado anteriormente indican que se ha pasado de un LCOE estimado para la generación eólica de $200 € / M W h$ en los años 80 a los niveles actuales señalados anteriormente de $52 € /$ MWh. El origen de dichas mejoras se puede encontrar en los siguientes factores:

1. Una reducción del coste de las turbinas desde más de 2 mill $€ / M W$ a menos de $900.000 € /$, entre la década de los 80 y hoy. 
2. Una muy relevante mejora en la eficiencia productiva de las nuevas máquinas, por combinación de mayor tamaño medio (MW por turbina), altura de torre, diámetro de barrido de pala y disponibilidad real.

3. La caída en el coste de O\&M a una quinta parte hoy respecto al registrado en los años 80 .

A estas ganancias no es neutral la creciente competencia entre fabricantes de turbinas, de un producto que tiene un importantísimo componente de innovación tecnológica, mejorando las prestaciones para permitir el aprovechamiento rentable de desarrollos anteriormente descartados, así como tampoco lo es la significativa mejora en la profesionalización y especialización de los servicios de O\&M.

Esta mayor competencia y necesidad de inversión en tecnología y recursos de conocimiento ha tenido, sin embargo, un significativo impacto sobre las tasas de rentabilidad de los fabricantes de turbinas, que han visto como sus márgenes operati$\operatorname{vos}^{13}$ han caído literalmente a plomo, desde niveles de $14-15$ por 100 a mediados de la pasada década a 2-4 por 100 en 2011. No obstante, en una situación de mercado normalizada es difícil esperar que dichos márgenes superen el 6-8 por 100, como indican algunos fabricantes en el sector ${ }^{14}$, lo que promete que seguiremos viendo nuevas mejoras tecnológicas y de costes en los productos, como única vía práctica para la supervivencia de estos fabricantes.

Analizados los costes y sus tendencias, toca ahora revisar el grado de competitividad frente a otras formas de generación convencionales, de combustible de origen fósil o nuclear.

En la obtención de sus LCOEs, hay dos variables neutrales para el cálculo del coste de la energía eólica, pero que tienen una influencia determinante en su competitividad relativa: el precio de los combustibles y el coste de las externalidades medioambientales. Nótese que, independientemente de los niveles actuales que tienen estas variables, un aspecto fundamental en este análisis es la visión que se pueda adoptar sobre sus tendencias a medio/largo plazo.

Adicionalmente la variable sostenibilidad medioambiental ha jugado (y esperemos siga jugando) un papel clave en el diseño de las políticas energéticas nacionales. En este sentido, la expectativa que el analista tenga acerca de los futuros requerimientos regulatorios o de desempeño técnico medioambiental de las tecnologías fósiles también va a jugar un papel en los niveles de competitividad relativos.

Todo este preámbulo para alertar al lector de que no es fácil llegar a conclusiones definitivas, blanco o negro, que las zonas grises son extensas y que, en definitiva, el análisis de la competitividad tiene casi tanto de arte como de ciencia.

¿Cuál es el coste de las energías convencionales? Los datos de un riguroso y exhaustivo estudio realizado por la Agencia Internacional de la Energía en $2010^{15}$

\footnotetext{
${ }^{13}$ Ratio EBIT/Ventas \%.

14 GAMESA Plan de Negocios 2011-2013.

15 AIE (2011): Projected Costs of Generating Electricity. 2010 Edition.
} 
ofrecían el siguiente panorama de costes estimados para plantas con puesta en operación en 2015 .

\section{GRÁFICO 5 \\ LEVELISED COSTS OF ENERGY PARA DISTINTAS TECNOLOGÍAS* (口/MWh)}

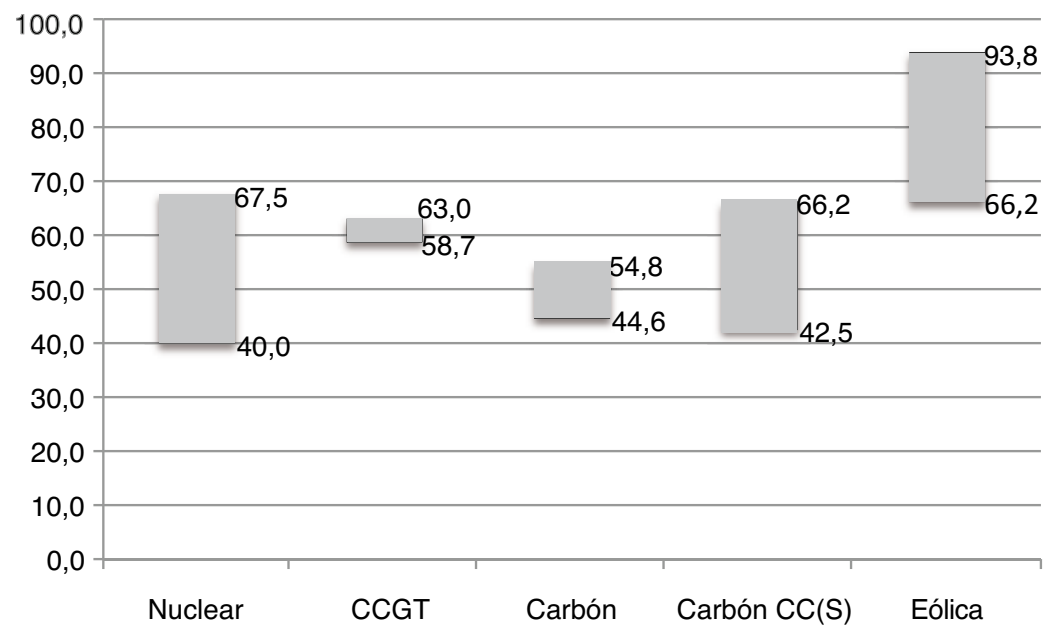

NOTA: * Medianas con tasas de descuento del 5 por 100 y 10 por 100 ; CC(S): considera los costes de instalar un equipo de captura de $\mathrm{CO} 2$, pero no de almacenamiento FUENTE: AIE (2011).

En primera instancia, de la lectura de estos datos se puede concluir que la energía eólica no sería competitiva sobre las bases estudiadas por la AIE. Sin embargo, con base en el análisis de BNEF para el segundo semestre de 2011 mencionado anteriormente, esta tecnología sí se encontraría dentro de un rango razonable de competitividad, al estimarse, recordemos, un LCOE de $52 € / \mathrm{MWh}$.

Por otro lado, y como decíamos anteriormente, incluso tomando estos datos de costes de la eólica de la AIE prima facie, no olvidemos la elevada sensibilidad que este tipo de análisis presenta ante distintos escenarios en las variables clave. Por ejemplo:

- Costes de $\mathrm{CO}_{2}$. La AIE ha utilizado un precio de $30 \mathrm{US} \$ /$ tn para los cálculos. Cada 1 US\$/tn de variación tiene un impacto de 1,0 US\$/MWh en los cash costs de las plantas de carbón (sin $\mathrm{CC}$ ). El análisis de sensibilidad realizado en el trabajo indica que un 50 por 100 de variación en el precio del CO2 supone una variación del 18 por 100 sobre el LCOE de las plantas de carbón.

- Los costes de inversión. Recordemos que para la eólica representan el 75 por 100 del valor del LCOE; en el caso del análisis de la AIE se utiliza una cifra 
de costes de inversión un 25 por 100 superior a los realmente registrados en desarrollos eólicos más recientes.

Si además incluimos los costes de equipos para el cumplimiento de niveles medioambientales más estrictos, el panorama cambia sustancialmente. En este sentido vale la pena consultar el informe encargado por Department of Energy \& Climate Change (DECC) del Reino Unido en agosto de $2011^{16}$, en el que se realizaba una estimación de los costes de plantas de generación convencional avanzadas futuras del tipo first of a kind (FOAK) y next of a kind (NOAK).

\section{GRÁFICO 6 \\ LEVELISED COSTS OF ENERGY PARA DISTINTAS TECNOLOGÍAS NOAK ( $\square /$ MWh)}

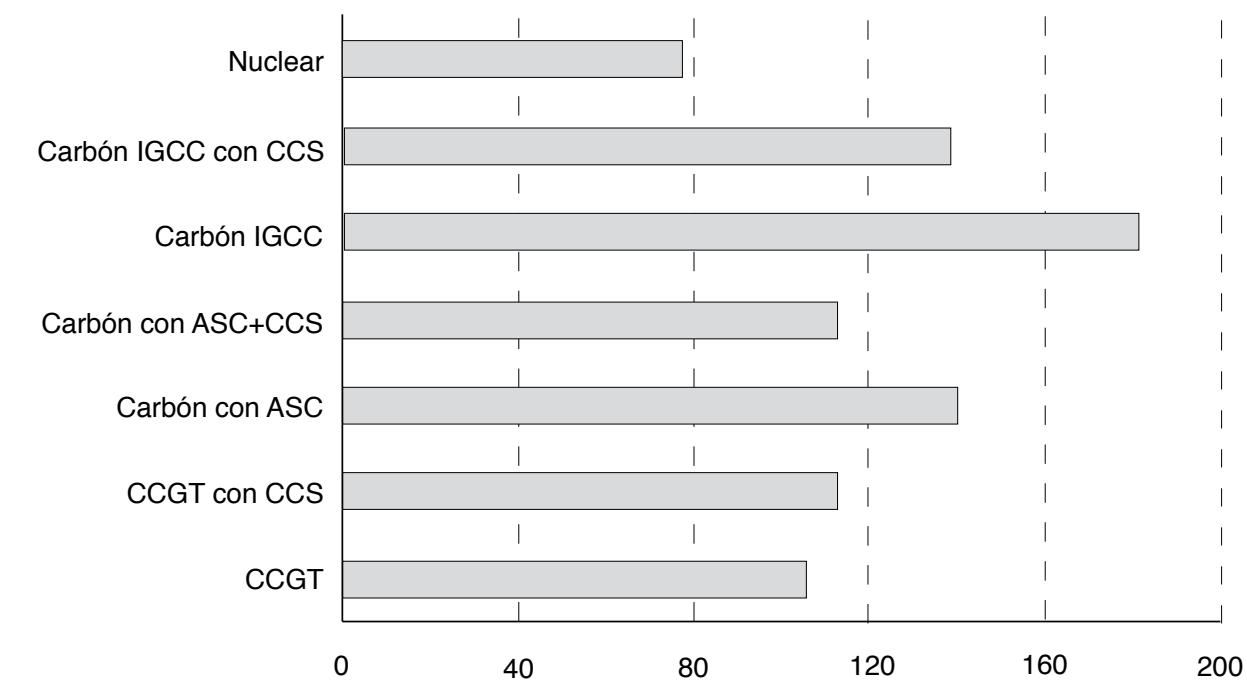

NOTA: ASC: Advanced Super Critical. CCS: equipo de captura y almacenamiento de $\mathrm{CO}_{2}$. Proyectos con fecha de comienzo de construcción en 2017

FUENTE: $\operatorname{DECC}$ (2011a), p. 17.

Como puede verse, este contexto de política energética orientada a combatir las externalidades medioambientales, haría a la electricidad de origen eólica plenamente competitiva sin necesidad de diseñar esquemas regulatorios de soporte específicos.

Finalmente, y por reforzar algunas de las ideas expuestas acerca de la importancia que tiene la consideración de las externalidades en el análisis de la competitividad de la energía eólica, el informe especial del Intergovernmented Power on Climate Change (IPCC) sobre fuentes de energía renovables ${ }^{17}$ presenta una interesante tabla

${ }^{17}$ IPCC (2011): Special Report on Renewable Energy Sources and Climate Change Mitigation. 
sobre el valor económico de las externalidades para distintos tipos de tecnologías de generación ${ }^{18}$, en el que la eólica aparece como la que menor nivel de externalidades produce, con una muy gran diferencia respecto al resto (renovables o no).

\section{GRÁFICO 7}

\section{VALOR ECONÓMICO DE LAS EXTERNALIDADES EN LA GENERACIÓN ELÉCTRICA (cUS\$/KWh)}

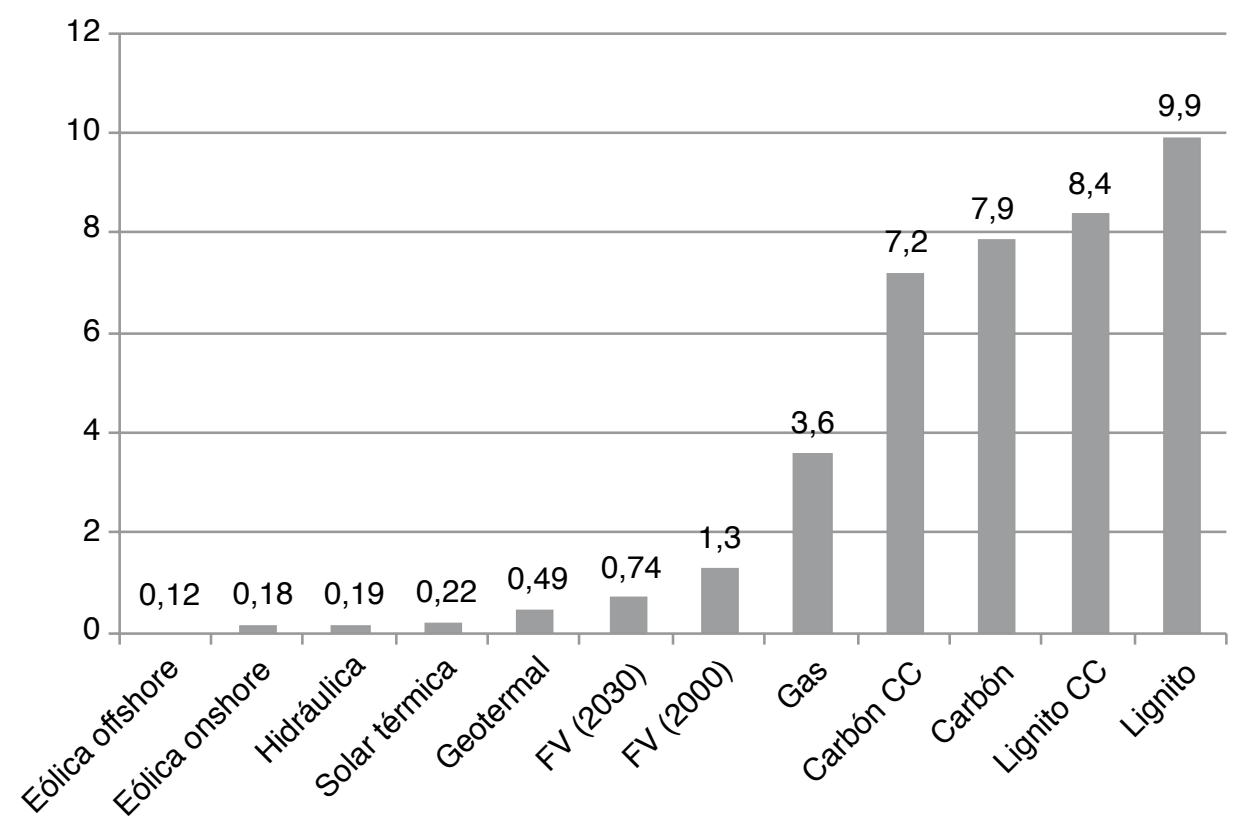

FUENTE: IPCC (2011).

\section{La nueva frontera: eólica marina}

La energía eólica de origen marino (offshore) existe desde 1991, cuando se instalaron las primeras 11 turbinas en un desarrollo frente a la costa de Dinamarca con una potencia total de 4,95 MW.

Pero sólo muy recientemente se ha empezado a hablar seriamente de esta tecnología que, debe reconocerse, aún está en fase temprana de desarrollo. De hecho a finales de 2010 la mayor parte de la potencia instalada se concentraba en Europa, con apenas $2.946 \mathrm{MW}$, indicando las primeras estimaciones de 2011 que se habrán alcanzado alrededor 3.800 MW. De estos, unos 2.100 MW están en Reino Unido,

\footnotetext{
${ }^{18}$ IPCC (2011), p. 855.
} 
algo más de 850 MW en Dinamarca y el resto en los Países Bajos, Alemania, Suecia y casi testimonialmente en Finlandia, Irlanda, Noruega y Portugal.

La eólica marina se encuentra por tanto en una situación de despegue, pero con un gran potencial futuro por delante. De hecho es la gran apuesta tecnológica de algunos de los principales fabricantes mundiales de turbinas eólicas como Siemens, Vestas o Gamesa

En esta sección del artículo vamos a dar algunas ideas a tres cuestiones clave:

- ¿Dónde se concentra el potencial de desarrollo y cuando se va a materializar?

- ¿Cuáles son las principales barreras para el desarrollo de la eólica marina?

- ¿Cuáles son sus costes y qué se está haciendo para reducirlos?

\section{¿Dónde se concentra el potencial de crecimiento?}

La apuesta por la energía eólica marina se encuentra muy focalizada en la región norte de Europa, destacando los importantes objetivos establecidos en Reino Unido (18 GW en 2020). En Alemania se podrían alcanzar $25 \mathrm{GW}$ en 2030 y en Francia unos $6 \mathrm{GW}$ en 2020.

Fuera del ámbito europeo, China (¡naturalmente!) se perfila en estos momentos como el país que más apuesta por esta tecnología, estimando que se habrán alcanzado 5 GW en 2015 y previendo llegar a 30 GW en 2030. Estados Unidos también presenta un importante potencial ${ }^{19}$ para el desarrollo de potencia eólica marina, pero la estrategia energética nacional actual no está incentivando suficientemente dicha tecnología.

Europa es, por tanto, la región más prometedora a corto plazo para la eólica marina, cuyo desarrollo viene apuntalado por los objetivos de descarbonización de la economía europea, la decidida apuesta política del Reino Unido por esta tecnología y el abandono de la energía nuclear por Alemania.

\section{¿Cuáles son las principales barreras para el desarrollo de la eólica offshore?}

1. Retos tecnológicos: la ingeniería de construcción de los parques eólicos en el mar está en una fase de work in progress en muchos aspectos, adquiriendo complejidad adicional a medida que los emplazamientos se alejan de la costa y deben abordarse nuevas técnicas de cimentación a gran profundidad.

La potencia unitaria de los aerogeneradores será mayor que la actual, y frente al estándar terrestre actual de 2,5MW, los marinos se moverán inicialmente entorno a los 5MW, para ir progresivamente escalando hasta los $7 \mathrm{MW}$ e inclu-

\footnotetext{
${ }^{19}$ El potencial teórico fue estimado en $830 \mathrm{GW}$ por el National Renewable Energy Laboratory (NREL) en un análisis publicado en junio de 2010
} 
so los 10MW, con la complejidad consiguiente para el transporte e instalación por el mayor tamaño y peso.

A lo anterior se unen unas condiciones de operación en el mar más complejas, con los materiales sujetos a fuertes procesos de corrosión. Esto planteará la necesidad de buscar nuevos diseño de torres, materiales y pinturas, especialmente para las palas, que garanticen altos niveles de disponibilidad del equipo generador.

2. Cadena de suministro. En paralelo a los retos tecnológicos descritos, nos encontramos también con una cadena de suministro también in progress, en forma de infraestructuras portuarias, naves para transporte y construcción de infraestructuras, o el adecuado dimensionamiento industrial para suministrar todo el material eléctrico necesario (cables, transformadores, etc).

3. Aspectos regulatorios: especialmente en estos primeros momentos de su lanzamiento, para una conseguir atraer los volúmenes de inversión esperados, se requerirá un marco regulatorio que garantice la mayor certidumbre posible sobre los flujo de ingresos, facilitando la financiación de largo plazo necesaria por este tipo de proyectos.

En algunos países como el Reino Unido, los mecanismos para la obtención de los permisos y licencias necesarios se ha revelado como una de las principales barreras para el desarrollo de los proyectos.

4. Desarrollo de las redes de transporte. A diferencia de los parques en tierra, los marítimos requieren el desarrollo de una costosa y compleja infraestructura de interconexión, que debe además ser construida en tándem con el propio parque, normalmente por un tercero distinto al promotor.

Hay por tanto la necesidad de coordinar no sólo distintos agentes económicos, sino también hacerlo con los organismos de planificación y el regulatorio correspondientes, sin olvidar que las infraestructuras de redes están sujetas a los mismos problemas de obtención de permisos que los discutidos anteriormente para los propios parques.

\section{¿Cuáles son los costes de la energía eólica marina?}

El perfil de costes de inversión de las instalaciones eólicas marinas es significativamente distinto del analizado en el caso de las terrestres. 


\section{GRÁFICO 8 \\ COSTES DE INVERSIÓN DE UN DESARROLLO EÓLICO MARINO (\%)}

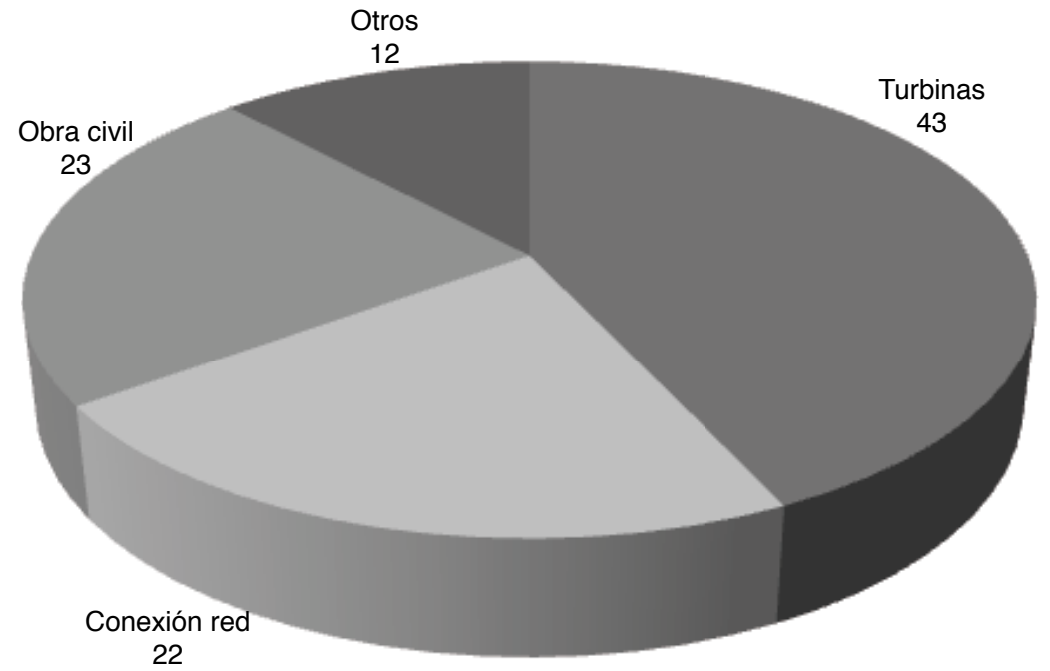

FUENTE: IPCC (2011).

Las turbinas representan entre un 40-50 por 100 de la inversión, frente al 75 por 100 en el caso de la eólica terrestre. Sin embargo los costes por MW instalado son significativamente superiores a las instalaciones eólicas terrestres, con estimaciones entre los 3,2-5,0 Mill US\$/MW ${ }^{20}$, esperando incluso que aumenten en el futuro ante la complejidad de los desarrollos en aguas profundas.

Por su lado, las estimaciones realizadas para los costes de O\&M multiplican en varias magnitudes a los de la eólica terrestre, habiéndose reportado para los parques actualmente en funcionamiento entre los 20 y 40 US\$/MWh.

La mayor producción esperada de estos emplazamientos (factores de carga de 35-45 por 100 medidos en el Reino Unido) no compensa en términos de los costes unitarios de energía el importante monto de las inversiones. Además, estos factores de carga pueden verse limitados por las disponibilidades reales que den las máquinas, que serán emplazadas en áreas de gran adversidad meteorológica durante buena parte del año.

En estas condiciones, el análisis realizado por IPCC $(2011)^{21}$ estima un rango de LCOE de 100-200 US\$/MWh. Un informe del National Renewable Energy Laboratory (NREL) de los Estados Unidos da un amplísimo rango de valores, jugando con distintos niveles del coste de inversión y de las tasas de descuento: 110-400 US\$/

\footnotetext{
${ }^{20}$ IPCC (2011): Special Report on Renewable Energy Sources and Climate Change Mitigation, p. 587.

${ }^{21}$ IPCC (2011): Special Report on Renewable Energy Sources and Climate Change Mitigation, p. 588.
} 
$\mathrm{MWh}^{22}$. El informe del Department of Energy and Climate Change (DECC) del Reino Unido ${ }^{23}$ ofrece un rango estimado de 149-191 GBP/MWh.

En definitiva, la energía eólica marina no es en estos momentos competitiva con alternativas convencionales o con la eólica terrestre. Sin embargo, esperamos que el proceso de mejora ya visto en esta última se replique en la eólica marina, y quizás con mayor velocidad dado el conocimiento y la experiencia adquirida en los últimos años.

Las políticas públicas no son neutrales en este esfuerzo para acelerar la curva de aprendizaje. Un ejemplo relevante lo está dando el Reino Unido, con diversas medidas de apoyo directo e indirecto para el desarrollo de la eólica marina en el país, pero con un claro objetivo también de minimizar el coste para los consumidores. Uno de los proyectos más relevantes ha sido la creación de una comisión técnica ${ }^{24}$, formada por expertos sectoriales de la industria, que debe presentar una hoja de ruta sobre cómo alcanzar un coste de producción (LCOE) de la eólica marina de 100GBP/ MWh en 2020.

\section{Conclusiones}

1. La pasada década ha sido testigo del despegue de la energía eólica y su consolidación como tecnología de generación mainstream, alcanzando una potencia instalada mundial de $238 \mathrm{GW}$ a finales de 2011. El desarrollo de esta potencia se ha dado inicialmente en los principales países desarrollados, pero en los dos últimos años se está produciendo un claro movimiento de traslación hacia las regiones en desarrollo, habiendo tomado el relevo China y, en un futuro no muy lejano, Brasil o India.

2. El marco regulatorio de apoyo a esta energía industria es clave para su desarrollo. Se han ensayado en estos años varios modelos, descritos en detalle en nuestro artículo, sin que haya una evidencia concluyente sobre el que presenta mayor efectividad. No obstante, sí se constata una mayor difusión de los mecanismos de primas a la producción y una gran presencia de modelos mixtos, que combinan dos o más mecanismos.

3. Gracias a un notable esfuerzo de inversión en investigación y a las economías de escala en la producción, los costes de la generación eólica terrestre han experimentado significativas mejoras en los últimos años y hoy pueden considerarse plenamente competitivos con otras alternativas de generación, tanto de base fósil como nuclear. Ello en el marco de unas políticas energéticas que sigan considerando la sostenibilidad medioambiental como uno de sus principales objetivos.

${ }^{22}$ NREL (2010): Large Scale Offshore Wind Power in the United States

${ }^{23}$ DECC (2011c): UK Renewable Energy Roadmap, p. 17.

${ }^{24}$ Offshore Wind Cost Reduction Taskforce. 
4. Finalmente realizamos un análisis de la energía eólica marina, que se perfila como el futuro campo de crecimiento para esta industria. Esta tecnología se encuentra en una fase temprana de desarrollo, enfrentada a retos importantes de carácter ingenieril y operativo, y presentando aún nivel de costes de producción muy elevados. No obstante, la traslación de las mejores prácticas desarrolladas en la eólica terrestre y la fuerte apuesta de algunos países clave europeos (Reino Unido o Alemania) permite ser relativamente optimistas sobre su curva de aprendizaje y la velocidad para constituirse en alternativa viable en el medio plazo.

\section{Referencias bibliográficas}

[1] AGENCIA INTERNACIONAL DE LA ENERGÍA (2011): Projected Costs of Generating Electricity-2010 Edition.

[2] DEPARTMENT OF ENERGY \& CLIMATE CHANGE (2011a): Electricity Generation Cost Model-2011 Update.

[3] DEPARTMENT OF ENERGY \& CLIMATE CHANGE (2011b): Planning Our Futurea White Paper for Secure, Affordable and Low-Carbon Electricity.

[4] DEPARTMENT OF ENERGY \& CLIMATE CHANGE (2011c): UK Renewable Energy Roadmap.

[5] EUROPEAN WIND ENERGY ASSOCIATION (2005): Support Schemes for Renewable Energy.

[6] EUROPEAN WIND ENERGY ASSOCIATION (2009): The Economics of Wind Energy.

[7] EUROPEAN WIND ENERGY ASSOCIATION (2011): Wind in Our Sails-The Coming of the European's Offshore Wind Energy Industry.

[8] EUROPEAN WIND ENERGY ASSOCIATION (2012): European Offshore Wind Industry-Key 2011 Trends and Statistics.

[9] GLOBAL WIND ENERGY COUNCIL (2012): Global Wind Statistics 2011.

[10] INTERGOVERNMENTAL PANEL ON CLIMATE CHANGE (2011): Special Report on Renewable Energy Sources and Climate Change Mitigation.

[11] NATIONAL RENEWABLE ENERGY LABORATORY (2010): Large-Scale Offshore Wind Power in the United States-Assessment of Opportunities and Barriers.

[12] SCHMALENSEE, R. (2011): Evaluating Policies to Increase the Generation of Electricity from Renewable Energy, Paper CEEPR 2011-008. 